\title{
Research on Extraction, Purification Isolation and Identification of Flavonoids from Mountain Chrysanthemum Leaves and the Antibacterial Characteristics
}

\author{
Lili Wang ${ }^{1}$ \\ ${ }^{1}$ Shijiazhuang People's Medical College, Shijiazhuang, Hebei, 050091 \\ hunter2011@foxmail.com
}

Keywords: Mountain Chrysanthemum Leaves; Flavonoids; Antioxidant; Hpyerglycemic; Bacteriostat

\begin{abstract}
This paper takes flavonoids the Mountain chrysanthemum leaves contained as the research object, using ultrasonic to extract total flavonoids and using response surface methodology to optimize flavonoids of mountain chrysanthemum leaves extraction process. The experiment use normal pressure silica gel column chromatography to isolate flavonoids, and take some tests to monomer and total flavonoids, for example of in vitro and in vivo antioxidant activity tests, the study of hypoglycemic activity and antibacterial activity etc. The purpose is to screen the active ingredient (monomers) of mountain chrysanthemum leaves.
\end{abstract}

\section{Introduction}

Extraction methods vary according to varied Ingredients arnica leaves, the unknown natural medicines for type identification of the chemical constituents of the system test methods (water extraction, alcohol extraction and petroleum ether extraction), and found that the water extracts detect a variety of ingredients proteins, amino acids, alkaloids, flavonoids, sugars, phenols and organic acids and saponins; petroleum ether extract detected coumarin and steroid and other ingredients; alcohol extract detection: Composition phenols, alkaloids, flavonoids and anthraquinones and organic acids initially speculated that the Kunlun chrysanthemum leaves, in addition to containing amino acids, sugars and flavonoids and saponins, there are proteins, organic acids, alkaloids, phenols, anthraquinone, steroids and coumarin and phenylpropanoids ingredients.

\section{Arnica Leaves Flavonoids Extraction Process Optimization}

Raw Materials and Equipment. Raw materials for the mountain daisy reagents, ethanol, aluminum nitrate, sodium nitrite, sodium hydroxide, petroleum ether AR;. Rutin standard GR 721-100 type visible spectrophotometer; SY-500 ultrasonic extraction device; SHZ-D ( III) circulating pumps; CP224C electronic balance; DZF-6000 electric vacuum oven; DZKW-5-4-type electric heated water bath; RE-5205 rotary evaporator

Experimental Methods. Take the required fine mountain chrysanthemum leaves powder was placed in $500 \mathrm{~mL}$ bottles extraction of petroleum ether, chloroform and ethyl acetate and n-butanol, adding petroleum ether degreasing, bleaching, chloroform extraction of small molecules, respectively; each extractant compartment $2 \mathrm{~h}$ and was repeated 3 times, collecting the extract was concentrated under rotation, recycling, chloroform, ethyl acetate, n-butanol same treatment, samples were dried for use.

Univariate Analytical Methods of Arnica Leaves Flavonoids. According to this study lot of information and documents related to the experimental nature of the study material, study the factors determining the use of ultrasonic-assisted extraction method for extracting arnica leaves flavonoids being chosen: extraction ultrasonic power, extraction volume percent ethanol, feed liquid ratio, extraction time, to inspect the condition of these four factors, respectively.

Liquid ratio Duishan chrysanthemum leaves flavonoids influence sperm extraction taking 1.000 g petroleum ether treated arnica leaf powder, said six copies, into $250 \mathrm{~mL}$ round bottom flask was 
added $80 \%$ ethanol extract ultrasonic power $50 \mathrm{~W}$ time $300 \mathrm{~min}$, solid-liquid ratio by 1: $5 \mathrm{~g} / \mathrm{mL}, 1$ : $10 \mathrm{~g} / \mathrm{mL}, 1: 15 \mathrm{~g} / \mathrm{mL}, 1: 20 \mathrm{~g} / \mathrm{mL}, 1: 25 \mathrm{~g} / \mathrm{mL}, 1: 30 \mathrm{~g} / \mathrm{mL}$, extracted. Must extract was filtered and set aside.

Ethanol concentration Duishan chrysanthemum leaves flavonoids influence sperm extraction taking 1.000 g petroleum ether treated arnica leaf powder 6 parts, into $250 \mathrm{~mL}$ round-bottomed flask, 1:20, extraction ultrasonic power $50 \mathrm{~W}$, extraction time $300 \mathrm{~min}$, the ethanol concentration of $10 \%, 20 \%, 40 \%, 60 \%, 80 \%, 100 \%$ at a temperature of $60{ }^{\circ} \mathrm{C}$ for extraction of $30 \mathrm{~min}$, the extract was filtered and set aside.

Extraction time on the mountain daisy leaves flavonoids extraction influence take-treated fine mountain daisy leaf powder 6 parts, into $250 \mathrm{~mL}$ round-bottomed flask 1.000 g petroleum ether, solid-liquid ratio 1: $20 \mathrm{~g} / \mathrm{mL}$, the concentration of ethanol $80 \%$ extraction ultrasonic power $50 \mathrm{~W}$, extraction time of $0 \mathrm{~min}, 10 \mathrm{~min}, 30 \mathrm{~min}, 50 \mathrm{~min}, 70 \mathrm{~min}, 90 \mathrm{~min}$, reflux extraction. The extract was filtered and set aside.

Extraction of ultrasonic power on the mountain daisy leaves flavonoids extraction of refined petroleum ether $1.000 \mathrm{~g}$ fetch fetch treated arnica leaf powder 6 parts each, into $250 \mathrm{~mL}$ round-bottom flask, solid to liquid ratio of $1: 20 \mathrm{~g} / \mathrm{mL}$, ethanol concentration $80 \%$, time 30 min, extraction ultrasonic power $10 \mathrm{~W}, 30 \mathrm{~W}, 50 \mathrm{~W}, 70 \mathrm{~W}, 90 \mathrm{~W}, 100 \mathrm{~W}$ power extraction under $30 \mathrm{~min}$. Get sample was filtered and set aside.

Based on the single factor experiment impact on arnica leaves flavonoids extraction rate were 4 factors and 3 levels of response surface optimization of single factor experiments, the best extraction process.

Refined to take $1.000 \mathrm{~g}$ treated arnica leaf powder, in response to surface testing to determine the optimal extraction conditions of extracting total flavonoids, and set the volume to $100 \mathrm{~mL}$ volumetric flask, with a pipette $2.00 \mathrm{~mL}$ were placed in five $10 \mathrm{~mL}$ flask, according to a standard curve absorbance values determined by the method to calculate the total flavonoid content.

Refined to take $1.000 \mathrm{~g}$ treated arnica leaf powder, in response to surface testing to determine the optimal extraction conditions of extracting total flavonoids, according to standard curve method, were measured six times every 10 min interval measured absorbance values to calculate the total flavonoids content.

Refined to take $1.000 \mathrm{~g}$ treated arnica leaf powder 5 parts in response to surface test to determine the optimum extraction conditions of extracting total flavonoids, do 6 sets of parallel experiments, according to the standard curve method for determining the absorbance values of total flavonoids formula.

Refined to take 1.000 g treated arnica leaf powder, 5 parts of each insertion of rutin standard solution, response surface optimization univariate analysis was to determine the optimal extraction conditions of total flavonoids extraction rate, the absorbance was measured according to the standard curve method rutin value recovery formula.

Refined to take $1.000 \mathrm{~g}$ treated arnica leaf powder, 5 parts of each insertion of rutin standard solution, response surface optimization univariate analysis was to determine the optimal extraction conditions of total flavonoids extraction rate, the absorbance was measured according to the standard curve method rutin value recovery formula.

\section{Separation and Purification of Arnica Leaves Flavonoids}

Materials, Reagents, Equipment and pre-treatment. Methanol CR; petroleum ether, chloroform, ethyl acetate, n-butanol, methanol, ethanol and other organic reagents AR; macroporous resin AB-8; silicone Model: 100-200 mesh RE-50B rotary evaporator: Shanghai Shanghai Health and Technology Co., BS124S electronic balance: Beijing Sartorius Instrument Systems, Inc., SWB-2000 water bath shaker: Tianjin Ott Sainz Instrument Co., SK3200H ultrasonic cleaning machine: Shanghai Branch of ultra ultrasound equipment company, macroporous resin were purchased in Anhui Samsung Resin Technology Co., Ltd.; Inova-400 NMR spectrometer: US company Varian, X-4 digital micro melting point apparatus, glass macroporous resin column $(\mathrm{d}=2$ 
$\mathrm{cm}, \mathrm{L}=30 \mathrm{~cm})$, glass silica gel column $(\mathrm{d}=2 \mathrm{~cm}, \mathrm{~L}=60 \mathrm{~cm}$ and $\mathrm{d}=5 \mathrm{~cm}, \mathrm{~L}=100 \mathrm{~cm})$.

Methods. Weigh accurately arnica leaf powder $1.000 \mathrm{~g}, 10 \mathrm{~mL}$ with ethanol sonication, filtered sample was dissolved with distilled water to $1000 \mathrm{~mL}$ volumetric flask to give concentration of 1.0 $\mathrm{mg} / \mathrm{mL}$ solution arnica flavonoids spare.

HPD-600, HPD-100, DM130, X-5, AB-8, D101 and other six kinds of different polarity macroporous resin for screening. Experimental selected tree as shown in Table 4-1, first with the amount of ethanol so that it can cover the resin above can be completely immersed, soak 24 h cause swelling of the resin is insufficient. Rinse and reach the resin no alcohol taste to stop, then wet packed with distilled water. Take good resin treated with 5\% HCI solution to soak the resin $6 \mathrm{~h}$, then washed with distilled water, i.e., a neutral $\mathrm{pH}$ of the effluent is detected before. Take good resin treated with $5 \% \mathrm{NaOH}$ solution immersion 6 h, then washed with distilled water, i.e., a neutral $\mathrm{pH}$ of the effluent is detected before. Cycle was repeated three times. Finally, the resin was completely soaked in distilled water pretreatment good backup. The $10.00 \mathrm{~kg}$ mountain chrysanthemum leaves crushed formulated using $1.5 \mathrm{~L} 80 \%$ ethanol in ultrasonic power $50 \mathrm{~W}$ ultrasonic extraction condition 3 times/30 min, filtration, combined filtrate recovery of ethanol was $80 \%$ ethanol extract thereof. The residue was washed with ethanol with ultrasonic waves under conditions of a concentration of $75 \%$ and then extracted 3 times $30 \mathrm{~min}$, combined filtrates were spin evaporated in vacuo and evaporated to give $75 \%$ ethanol extract solution, and then $80 \%$ ethanol extract was mixed with $75 \%$ ethanol extract, the resulting mixture the sample was dissolved in distilled water with a small amount can, in turn make four kinds of extraction agent extraction volume grading: petroleum ether, chloroform and ethyl acetate and n-butanol gradient extraction, each extraction agent and extracted three times the average for each extraction solution was concentrated under reduced pressure to give the four parts of the sample extract and the water was.

\section{Antibacterial Characteristics of Arnica Leaves Flavonoids}

Experimental Materials, Equipment and Reagents. Pyrogallol AR Kaifeng Chemical Reagent, crystal violet solution AR Po Shan Shanghai Chemical Co., Ltd., Tianjin AR salicylic chemical equipment factory, Tris Jinxin God Bless Co., ferrous sulfate solution AR Xi'an Chemical Reagent, H2O2AR Tianjin Reach Chemicals Co., DPPH: Japan and Wako Pure Chemical Industries, Ltd., ABTS (2,2'- hydrazine - bis (3-ethyl-benzo-thiazoline-6-sulfonic acid) diamine salt): Sigma-Aldrich Companies, Vc Tianjin Chemical Reagent Factory Dengfeng AR, AR ethanol Chemicals Co., Ltd. Tianjin Zhiyuan.

Experimental Methods. Crude flavonoids A chrysanthemum leaves the mountain with 70\% ethanol, at 15 times the amount of solvent, ultrasonic extracted three times, each time 10 min, have rotation to give arnica extract crude flavonoids leaf alcohol labeled A sample. with petroleum ether, chloroform, ethyl acetate, n-butyl alcohol solvent and the aqueous phase was extracted respectively different phase was extracted with chloroform extracts were respectively as B, The ethyl acetate solution of C, D n-butanol solution of the sample, the aqueous phase was separated by silica gel E. Sample F. Vc monomer substance concentration $2 \mathrm{mg} / \mathrm{mL}$

Arnica Flavonoids Scavenging Effect on Free Radicals. Each sample was weighed $20.00 \mathrm{mg}$ dissolved in $5 \mathrm{~mL}$ of ethanol, respectively, draw $1 \mathrm{~mL}$ sample was diluted to $5 \mathrm{~mL}$. In this way it is diluted 3 times, prepared concentrations were $2 \mathrm{mg} / \mathrm{mL}, 1.3 \mathrm{mg} / \mathrm{mL}, 0.43 \mathrm{mg} / \mathrm{mL}, 0.14 \mathrm{mg} / \mathrm{mL}$, the concentration gradient of $0.048 \mathrm{mg} / \mathrm{mL}$ of. Using pyrogallol autoxidation method, take the tube number added $50 \mathrm{mmoL} / \mathrm{LTris}-\mathrm{HCl}$ buffer $\mathrm{pH} 8.24 .5 \mathrm{~mL}$ of distilled water and $4.2 \mathrm{~mL}$, after mixing the reaction at room temperature for $20 \mathrm{~min}$, added $25 \mathrm{mmoL}$ immediately after removing/L phthalic Three phenol solution $0.3 \mathrm{~mL}$, poured into cuvette at $319 \mathrm{~nm}$ absorbance values measured at intervals of $30 \mathrm{~s}$ after the rapid shake A0 to 4min stop when calculating the added value of absorbance per minute. Sample tube before adding pyrogallol, before adding different concentrations of arnica flavonoids solution $1 \mathrm{~mL}$, distilled water to fill the measured absorbance A1, and replace with distilled water Pyrogallol measured sample background value A2. Let Vc same manner as a positive control. 
Each sample was weighed $20.00 \mathrm{mg}$ dissolved in $5 \mathrm{~mL}$ of ethanol, respectively, draw $1 \mathrm{~mL}$ sample was diluted to $5 \mathrm{~mL}$. In this way it is diluted 3 times to prepare concentrations of $4.0 \mathrm{mg} / \mathrm{mL}$, $1.3 \mathrm{mg} / \mathrm{mL}, 0.43 \mathrm{mg} / \mathrm{mL}, 0.14 \mathrm{mg} / \mathrm{mL}$, the concentration gradient of $0.048 \mathrm{mg} / \mathrm{mL}$ of. The lower the absorbance, indicating that the sample to remove $\cdot \mathrm{OH}$ better. Configuring salicylic acid concentration $9 \mathrm{mmoL} / \mathrm{L} ; \mathrm{FeSO}_{4}$ concentration $9 \mathrm{mmoL} / \mathrm{L}$, a concentration of $8.8 \mathrm{mmoL} / \mathrm{L} \mathrm{H}_{2} \mathrm{O}_{2}$.

Take five test tubes and sequentially numbered, each tube add $1 \mathrm{~mL}$ of each concentration 9 mmoL/FeSO4 and $2 \mathrm{~mL}$ concentration $9 \mathrm{mmoL} / \mathrm{L} \mathrm{L}$ of salicylic acid - ethanol solution, according to number each added $2 \mathrm{~mL}$ tubes with different concentrations of arnica leaf flavonoids solution was added $2 \mathrm{~mL}$ final concentration of $8.8 \mathrm{mmoL} / \mathrm{L}$ of $\mathrm{H}_{2} \mathrm{O}_{2}$, start a reaction at room temperature for 30 min, the measured absorbance A1. Salicylic acid with distilled water and replace with the same method, the measured $\mathrm{A}_{2}$ distilled water instead of arnica flavonoids solution as a blank zero, measuring the absorbance at $510 \mathrm{~nm}$ wavelength A0, record data. The same method Vc positive control.

\section{Conclusion}

In this thesis, arnica extract Flavonoids were vivo anti-oxidation experiments. It provides a theoretical basis for the development of pharmacological research depth chrysanthemum leaves mountain pure natural flavonoids Only in sufficient concentration of free radical scavengers, only the role of free radicals in the position can be played. Location natural flavonoids as free radical scavengers only when a sufficient concentration of free radicals generated and only takes effect when nearby. In addition, when react with free radicals, scavenger itself becomes a free radical, this new radical toxicity or inert should be less than the original free radical toxicity or inert to play a protective role. With the deepening of the concept of green food, diet is one of the most reliable method for the prevention and treatment of diseases, development of flavonoids and health drinks has broad market prospects.

\section{References}

[1] Sandra RG, Rbia CR, Valria MD. Ev Aluation of the Antioxidant Activity of Different flavonoids by The Chemiluminescence Method. AAPS Pharmsci, 2003, 5(2): 20-26.

[2] Nccook, Ssamman. Flavonoids-Chemistry, Metabolism, Cardioprotective Effects and Dietarysources. J Nutrbiochem, 1996, 7: 66-76.

[3] Rice-Evans, C. A. Miller, N. J. Bolwell, P. G.Bramley, P.M. Pridham, J. B. The Relative Antioxidantactivities of Plantderived Polyphenolic Flavonoids. Free Radical Res. 1995, 22, 375-383.

[4] Pietta, P. G. Flavonoids as Antioxidants. J. Nat. Prod. 2000, 63, 1035-1042. 